\title{
Phase Transition Lowering and Melting in Dynamically-Compressed Silicon and Germanium at the LCLS
}

\author{
Emma Elizabeth McBride \\ SLAC National Accelerator Laboratory, Menlo Park, United States of America; \\ emcbride@slac.stanford.edu
}

Despite being the subject of numerous shock compression studies, the behavior of silicon under dynamic loading is vigorously debated [1-4]. The few studies that combine shock compression and X-ray diffraction have exclusively focused on "normal" X-ray geometry whereby X-rays are collected along the shock propagation direction, consequently sampling numerous strain states at once, and hence greatly complicating both phase identification and studies of phase transition kinetics.[5] Here, we present a novel setup to perform in situ X-ray diffraction studies perpendicular to the shock propagation direction at the Matter in Extreme Conditions end station at Linac Coherent Light Source, SLAC. Combining the extremely bright, micro-focused X-ray beam available at the LCLS with a nanosecond laser driver, we unambiguously characterize of the complex multi-wave shock response in silicon for the first time. We further combine this platform for performing simultaneous imaging with X-ray diffraction from shock compressed germanium, revealing its behaviour following shock compression. We note the transverse geometry is significantly more sensitive to the onset of both solid-solid and solid-liquid phase transformations in materials which exhibit complex multi-wave behaviour, and compare and constrast the behaviour of $\mathrm{Si}$ and $\mathrm{Ge}$.

[1] Graham et al., JPCS, 27, 9 (1966)

[2] Turneaure \& Gupta, APL, 90, 051905 (2007)

[3] Colburn et al., JAP, 43, 5007 (1972)

[4] Gust \& Royce, JAP, 42, 1897 (1971)

[5] Turneaure et al., PRL,121, 135701 (2018)

Keywords: Extreme Conditions, Free Electron Lasers, Shock compression, Phase transitions 